\title{
Dynamic Analysis of the hFan, a Parallel Hybrid Electric Turbofan Engine
}

\author{
George L. Thomas ${ }^{1}$ \\ N\&R Engineering and Management Services, Inc., Parma Heights, OH, 44130, USA \\ Dennis E. Culley ${ }^{2}$, Jonathan L. Kratz ${ }^{3}$, and Kenneth L. Fisher ${ }^{4}$ \\ NASA Glenn Research Center, Cleveland, OH, 44135, USA
}

\begin{abstract}
NASA and a variety of aerospace industry stakeholders are investing in conceptual studies of electrified aircraft, including parallel hybrid electric aircraft such as the Subsonic Ultra Green Aircraft Research (SUGAR) Volt. At this point, little of the work published in the literature has examined the transient behavior of the turbomachinery in these systems. This paper describes a control system built around the hFan, the parallel hybrid electric turbofan engine designed for the SUGAR Volt concept aircraft. This control system is used to show that the hFan, running with its baseline concept of operations, is capable of transient operation throughout the envelope. The design parameters of this controller are varied to assess the amount of operability margin built into the engine design, and whether this margin can be reduced to enable more aggressive designs, that may feature better fuel economy. Further, studies are performed as parameters for the hFan electric motor are varied to determine how the motor impacts the engine's need for transient operability margin. The studies suggest that the engine may be redesigned with as much as a $3 \%$ reduction in high pressure compressor stall margin. It was also demonstrated that appropriate design and control of the electric motor may be able to buy an additional $0.5 \%$ stall margin reduction or a turbine inlet temperature reduction of $35{ }^{\circ} \mathrm{R}$, as tested at the sea-level static condition.
\end{abstract}

$\begin{array}{ll} & \\ \text { CONOPS } & \text { Concept of operations } \\ \text { CRZ } & \text { Cruise } \\ \text { CV } & \text { Control variable } \\ \text { EPC } & \text { Electrical power code } \\ \text { FAR } & \text { Fuel-to-air ratio } \\ \text { Fn } & \text { Net thrust, lbf } \\ \text { HPC } & \text { High-pressure compressor } \\ \text { HPT } & \text { High-pressure turbine } \\ \text { IWP } & \text { Integral windup protection } \\ \text { LPC } & \text { Low pressure compressor } \\ \text { LPT } & \text { Low pressure turbine } \\ \text { MCL } & \text { Maximum climb } \\ \text { MCR } & \text { Maximum cruise } \\ \text { MEA } & \text { More electric aircraft } \\ \text { MIMO } & \text { Multi-input, multi-output } \\ \text { MTO } & \text { Maximum take-off } \\ \text { NO } & \text { Nitrogen oxides } \\ N 1 & \text { Low pressure shaft speed, rpm } \\ N 2 & \text { High pressure shaft speed, rpm }\end{array}$

${ }^{1}$ Controls Engineer, Intelligent Control and Autonomy Branch, george.l.thomas@nasa.gov

${ }^{2}$ Research Engineer, Intelligent Control and Autonomy Branch, dennis.e.culley@nasa.gov

${ }^{3}$ Research Engineer, Intelligent Control and Autonomy Branch, jonathan.kratz@nasa.gov

${ }^{4}$ Research Engineer, Propulsion Systems Analysis Branch, klfisher@nasa.gov 


$\begin{array}{ll}N 1 R & \text { Corrected fan speed, rpm } \\ \text { NPSS } & \text { Numerical propulsion system simulation } \\ \text { N+3 } & 3^{\text {rd }} \text { generation technology from current generation } \\ \text { Op-line } & \text { Operating line (on a turbomachinery performance map) } \\ \text { PC } & \text { Power code } \\ \text { PI } & \text { Proportional-Integral } \\ \text { Pm } & \text { Motor power, hp } \\ \text { PR } & \text { Pressure Ratio } \\ P s 3 & \text { Static pressure at station } 3 \text { (high pressure compressor exit), psi } \\ \text { RTO } & \text { Rolling take-off } \\ \text { RU } & \text { Ratio-Unit (specifically, Wf/Ps3) } \\ \text { SISO } & \text { Single-input, single-output } \\ \text { SLS } & \text { Sea level static (0 feet altitude and 0.0 Mach number) } \\ \text { SM } & \text { Surge margin, } \% \\ \text { SMA } & \text { Shape memory alloy } \\ \text { TO } & \text { Take-off } \\ \text { TOC } & \text { Top of climb } \\ T r & \text { Acceleration response time } \\ \text { TSFC } & \text { Thrust specific fuel consumption } \\ \text { TTECTrA } & \text { Tool for Turbine Engine Closed-loop Transient Analysis } \\ T 4 & \text { Temperature at station } 4 \text { (high pressure turbine inlet), }{ }^{\circ} \text { R } \\ \text { VBV } & \text { Variable bleed valve } \\ \text { VAFN } & \text { Variable area fan nozzle } \\ W f & \text { Fuel flow, lbm/s } \\ W f / P s 3 & \text { Control limiter, fuel flow divided by high pressure compressor static pressure } \\ & \end{array}$

\section{Introduction}

$\mathrm{T}$ HE aerospace industry has begun evaluating a variety of more electric aircraft (MEA) concepts and technologies, as a means of increasing mobility as part of NASA's Strategic Implementation Plan, improving system efficiency, noise, and/or emissions, as well as a means to add new capabilities to aircraft. These means include electrically driven fuel pumps and actuators ${ }^{1}$ that replace conventional (hydraulic or mechanical) ones. It also includes electric machines that provide new functionality that enables hybrid electric or turboelectric propulsion systems. ${ }^{2}$ NASA has contributed to the planning and research on MEA by setting aggressive goals for $\mathrm{N}+3$ subsonic aircraft as well as performing and coordinating research towards meeting these goals. N+3 refers to aircraft and associated technologies targeted for three generations beyond the state of the art. The $\mathrm{N}+3$ goals include a $71 \mathrm{~dB}$ reduction in noise, $75 \%$ reduction in nitrogen oxides $\left(\mathrm{NO}_{\mathrm{x}}\right)$ emitted during landing and takeoff, and $70 \%$ reduction in aircraft fuel burn compared to a single aisle reference aircraft based on the Boeing 737-800 with CFM56-7B engines. ${ }^{3}$ This paper focuses on dynamic analysis of the $\mathrm{N}+3 \mathrm{hFan}$ engine, which is a turbofan with an integrated motor connected to the low pressure shaft, as designed for the Subsonic Ultra Green Aircraft Research (SUGAR) Volt aircraft. ${ }^{4}$

The remainder of the paper is as follows. Section II provides a definition of the hFan model, and Section III discusses a study that was performed on the open-loop engine model to discover relationships between inputs and outputs. Section IV describes development of a control system for the hFan as well as some studies performed to ensure that the resulting closed-loop system is operable throughout the flight envelope. Finally, Section V presents results of dynamic systems analysis trade studies that explore trends in the relationships between transient operation and the necessary steady-state operability constraints, and Section VI provides concluding remarks and suggests directions for future work.

\section{NASA hFan Engine Model Definition}

The NASA hFan is a Numerical Propulsion System Simulation (NPSS) ${ }^{5}$ model of a hybrid electric 17,000 lbf thrust class two spool turbofan. It features an electric motor mounted in the engine tail cone that is connected to the low pressure shaft, and a variable area fan nozzle (VAFN). An S-function interface is available for NPSS, which allows an NPSS model to be run transiently within a MATLAB/Simulink model. This enables integration of NPSS engine models (i.e., engine plant models) into a closed-loop control system, for purposes of developing and analyzing engine controls. ${ }^{6}$ The NPSS S-function interface allows the actual NPSS engine model to run within Simulink, with

American Institute of Aeronautics and Astronautics 
its inputs and time variable controlled via Simulink and its outputs sent back to Simulink. A notional schematic showing hFan components related to engine controls is shown in Figure 1.

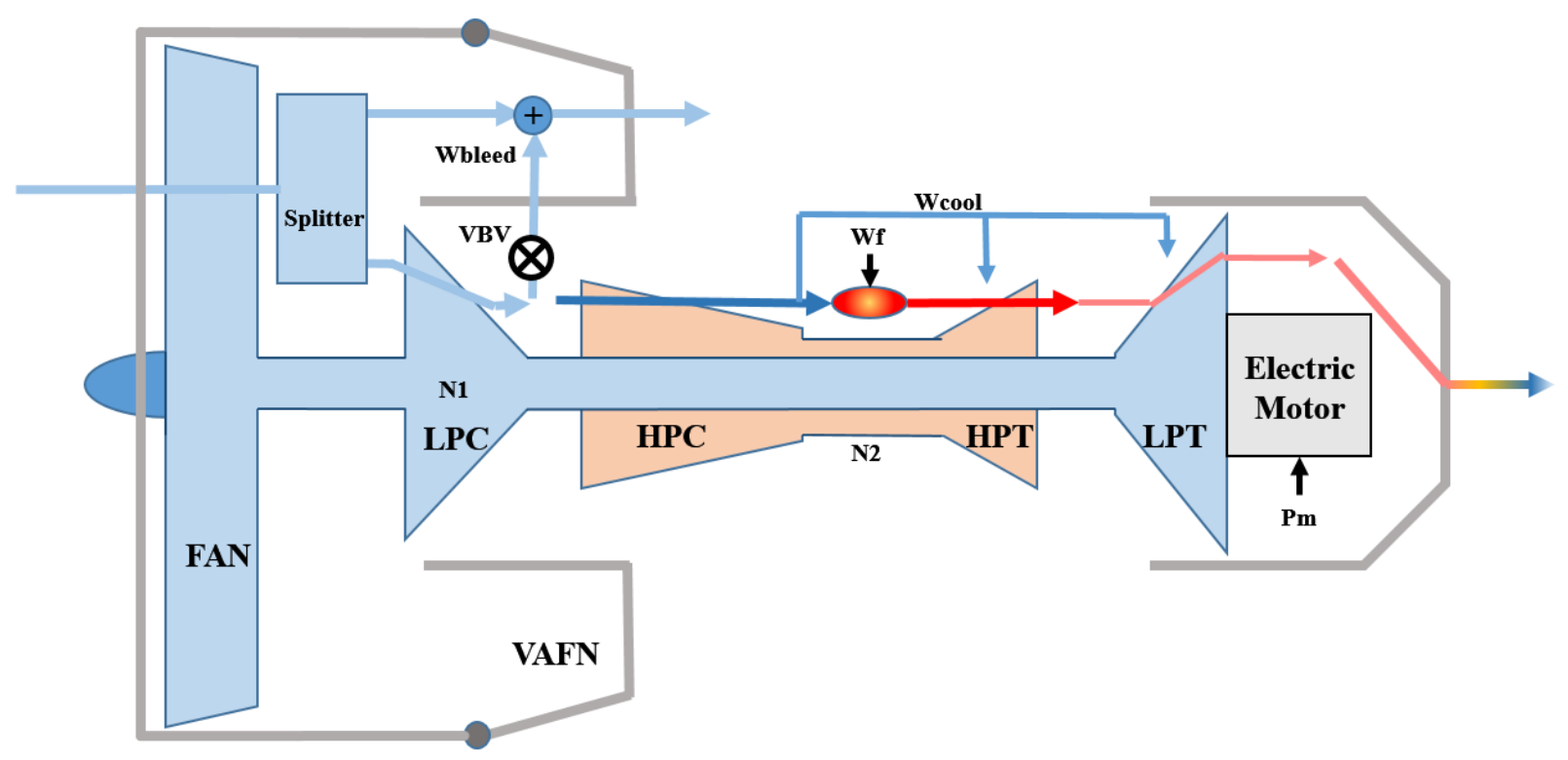

Figure 1: Schematic of hFan propulsion system, showing the turbomachinery components (the fan, LPC, HPC, HPT, and LPT), as well as the control inputs (VBV, $W f$, and Pm).

As indicated by this diagram, the $\mathrm{hFan}$ has a low pressure shaft which connects the fan, the low pressure compressor (LPC), and the low pressure turbine (LPT). The high pressure compressor (HPC) and high pressure turbine (HPT) are connected via the high pressure shaft. The low pressure and high pressure shaft speeds are denoted NI and $N 2$, respectively. The hFan turbomachinery components are modeled via performance maps that define the relationships between corrected speed, pressure ratio, corrected mass flow rate, and thermodynamic efficiency for each component. The majority of the power for the engine is supplied via combustion of fuel that is added to the burner, with a specified mass fuel flow rate $(W f)$. However, the $\mathrm{hFan}$ also features an electric motor that is mounted directly on the low pressure shaft and is used to augment thrust during various operating conditions.

A flow splitter component is included after the fan in this model. The splitter diverts the majority of the fan flow to the bypass duct and allows the rest to flow through the core, achieving a bypass ratio design value of $18: 1$. The bypass flow exits through the variable area fan nozzle (VAFN), which is controlled to set up the desired fan operating line, or op-line. The variable bleed valve (VBV) allows flow to be diverted from the back of the LPC to the bypass duct to control the LPC stall margin.

The hFan electric motor is designed with a power rating of $1380 \mathrm{HP}$ (approximately $1 \mathrm{MW}$ ). This motor is intended to be driven by battery power and to produce mechanical shaft power that varies with thrust demand. Note that the motor is designed to run at varying, nonzero power levels for the entirety of each flight mission. This mode of operation is consistent with the original hFan study flight operation for the SUGAR Volt engine from Ref. [7]. Also, this hFan model is designed to the same specifications as the " $\mathrm{hFan}+2750$ balanced" configuration ${ }^{7}$. The "750" part of the designation refers to the $750 \mathrm{Wh} / \mathrm{kg}$ battery energy density technology assumption. The "balanced" part comes from the fact that the engine was designed for a concept of operations (CONOPS) where between 10 and 15 percent of the fan shaft power comes from the motor at the maximum power setting, and the remaining power comes from the LPT via fuel flow. This is considered balanced compared to the design case where the motor is sized to allow the core/combustor to shut down and the fan to be driven solely by the motor at cruise. The dynamics for the motor, as well as the VAFN and VBV are all represented by first order transfer functions with saturation that enforces the actuator minimum and maximum limits.

For the purposes of control design and analysis, the CONOPS for the hFan was defined via (steady-state) engine thrust requirements. These are the thrusts required by the SUGAR Volt airframe design for different flight segments or modes such as takeoff (TO), rolling takeoff (RTO), top-of-climb (TOC), and cruise (CRZ). These thrust requirements map to power or throttle settings such as maximum takeoff (MTO), maximum climb (MCL), maximum

American Institute of Aeronautics and Astronautics 
cruise (MCR), and flight idle. In the hFan model in Ref. [7], the engine power setting is specified by variables called power code (PC) and electric power code (EPC). PC varies from 21 to 50, and corresponds to the total thrust produced by the engine (a PC value of 50 corresponds to the maximum power that the engine is capable of producing, and a PC value of 21 corresponds to a minimum idle power setting). EPC varies from 0 to 50 , and it corresponds to the fraction of rated mechanical power delivered by the motor to the low-pressure shaft, with an EPC value of 50 being the full rated power, and an EPC value of 0 corresponding to a motor power of 0 .

In this work, instead of using PC and EPC, the total power level input variable for the closed-loop controller is defined as a fraction of maximum thrust ( 0 being zero thrust, 1 being maximum thrust attainable at the given flight condition). For the propulsion system, this is referred to in this paper as the "thrust setpoint" or "setpoint" and is chosen because it is well suited for testing thrust response time. To meet the same thrust requirements and power splits set out in Ref [7], a steady-state relationship between the fraction of maximum thrust command and motor power (Pm) schedule was determined. This relationship is shown in Fig. 2.

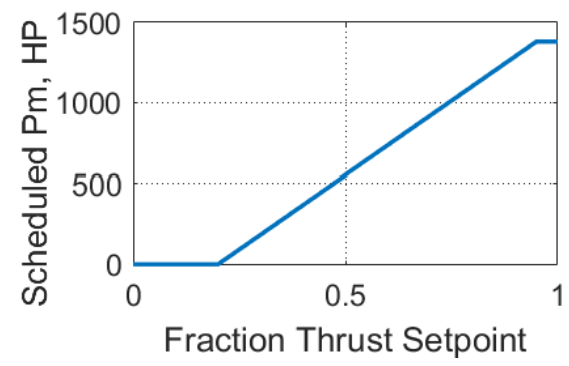

Figure 2: Relationship between thrust setpoint and motor power (Pm)

Given this engine system definition, the hFan simulation was integrated into Simulink via the NPSS S-Function and set up to run open-loop simulations to begin assessing the engine's dynamic response.

\section{Open-Loop Study}

Before attempting to create a closed loop controller, it was desired to do a qualitative, open-loop study to determine how the electric motor affects the system. A motor power step transient was applied at different thrust setpoint conditions, with the engine at sea-level static, to determine how the turbomachinery components behave when power is added to the low spool. For these transients, Pm was increased from its scheduled value at the given thrust setpoint (see Fig. 2) up to its maximum value of $1380 \mathrm{hp}$. Figure 3 shows the unscaled performance maps for each of the $\mathrm{hFan}$ components, along with the simulation data plotted on them.

American Institute of Aeronautics and Astronautics 


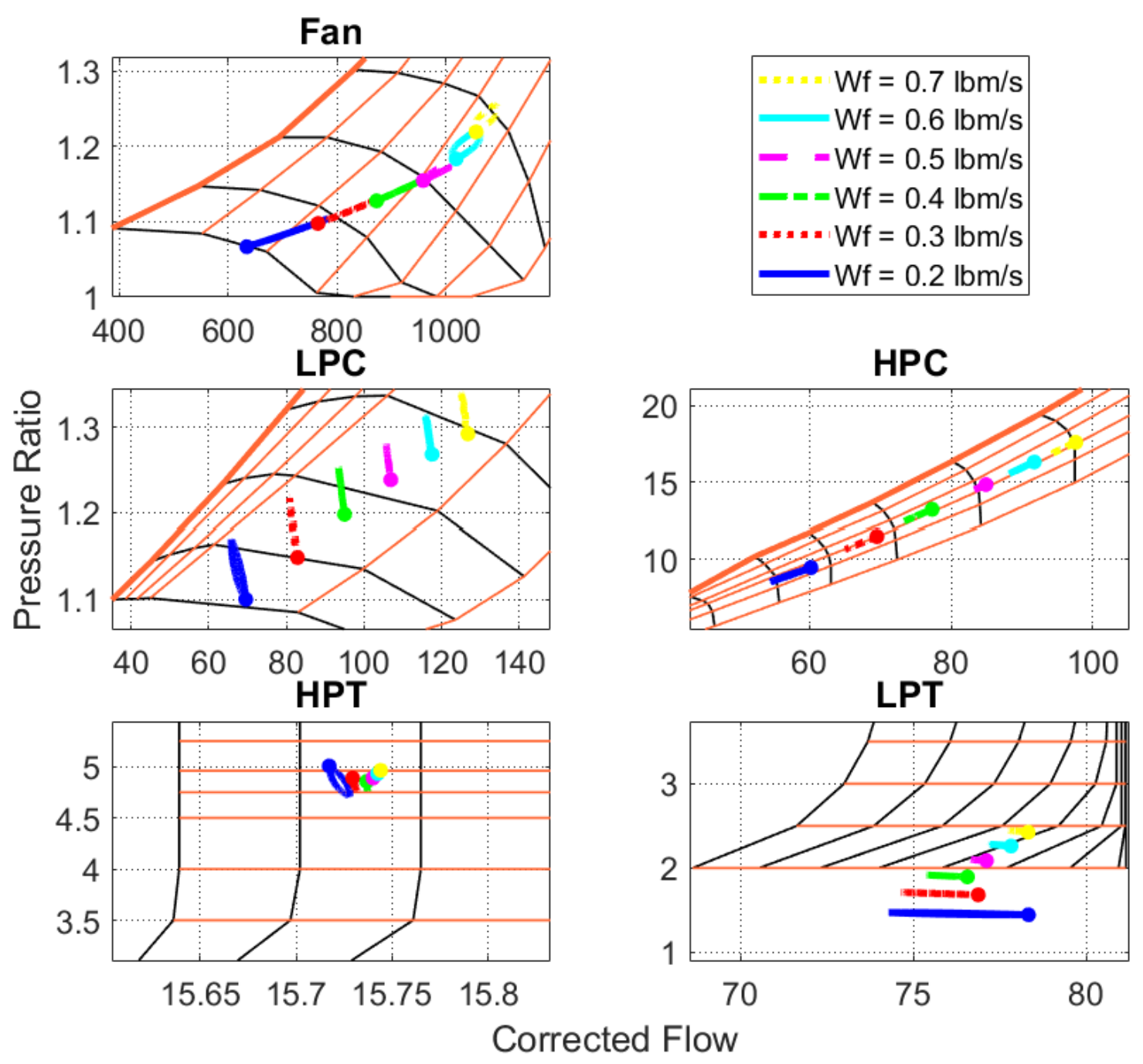

Figure 3: Plots of turbomachinery component performance maps, showing trajectories driven by motor power transients at different constant fuel flows.

In the figure, the data from each simulation begin with zero low spool motor power, and these initial points are indicated with dots for each of the simulations on each of the maps. The power is then stepped up and back down to observe how the components operate with power addition. Note that the orange lines correspond to R-lines for the compressors, and constant pressure ratio (PR) lines for the turbines, whereas the black lines correspond to constant corrected speed lines. The R-line variable is a dummy parameter that is used for parameterizing NPSS compressor performance maps. R-line is useful, because each combination of R-line and speed will yield a single unique value for parameters such as PR, corrected flow, and component efficiency. Note that the thick orange line on the compressor maps is the stall line. It is common in NPSS to define the stall line as R-line $=1$, and it is common that the value of the R-line parameter increases as the operating point of interest gets further and further away from the stall line.

The stall line is a line on the compressor map that separates the region of normal operation (below the line) from the region where compressor stall is likely to occur (above the line). Given that stall is a negative phenomenon, it is desirable to design the engine such that the components always operate an ample distance from the stall line, including during transients and as the engine's performance varies throughout its lifespan. The distance between a compressor's current operating point and its stall line, often expressed as a percentage, is called compressor stall margin (SM). Note that, given that an engine's turbomachinery components are coupled via the airflow through the engine, the compressor op-lines and thus stall margins that an engine will exhibit at steady-state are a consequence of the system-level engine design. In order for engine designs to be feasible, their op-lines must be placed such that they operate with enough

American Institute of Aeronautics and Astronautics 
stall margin to account for uncertianties (known as the uncertianty stack or stack-up), in addition to enough margin to account for losses during engine power or thrust setting transients (known as the transient stack). In this work, it is assumed that $10 \%$ stall margin must be maintained at all times for the uncertianty stack, and dynamic analysis is performed to determine what appropriate level of stall margin should be maintained at steady-state to represent the transient stack.

The major takeaways from the data in Figure 3 are that power addition onto the low spool (while holding the fuel flow constant) increases the low spool corrected speed, and slightly decreases the high spool corrected speed. Note that most of the change in high spool corrected speed occurs because of changes of the HPC inlet conditions, and not because of changes to the high spool mechanical speed. These corrected speed changes, in turn, affect the energy balance in the engine such that the LPC PR increases and LPT corrected flow decreases. At the same time, the fan is pushed up along its op-line, the HPC is pushed downward along its op-line, and the HPT is largely unaffected. The fact that the LPC PR is affected by the power addition suggests that a low spool motor offers some degree of LPC stall margin control. This also suggests that it may be deleterious in terms of LPC SM to run the motor at low LPC corrected speeds (low thrust setting) as higher motor power corresponds to a lower LPC SM. This information was found to be invaluable when designing the closed-loop system. Lastly, at low fuel flow settings at this flight condition, the LPT runs off of its map with a low pressure ratio. Fortunately, NPSS extrapolates the LPT map, and the simulation still produces reasonable results when this occurs.

\section{Closed-Loop Study}

\section{A. Closed-Loop Controller Design Test}

As was done in previous work, ${ }^{8}$ the open-source Tool for Turbine Engine Closed-loop Transient Analysis (TTECTrA) controller architecture and design tools were used to close the loop on the engine plant (the hFan). One of the goals of this work is to identify any possible transient operability issues throughout the flight envelope with the $\mathrm{hFan}$ concept. To this end, it was desired to design a full envelope controller to demonstrate full envelope transient operability. Note that in previous studies using TTECTrA for dynamic systems analysis, controllers were only designed for the Sea Level Static (SLS) flight condition. ${ }^{8,9,10}$ However, TTECTrA is capable of designing controller parameters for use in a gain-scheduled, full envelope controller, and so this is the approach taken in this work. Further, it was deemed sufficient to design a single-input, single-output (SISO) thrust control loop for the fuel flow actuator, and treat the motor power delivered as a disturbance to this controller. This is reasonable because the motor is rated to supply less than $15 \%$ of the low spool shaft power.

In this work, the net thrust output from the engine is assumed to be a quantity that is possible to estimate accurately, and this thrust output is used as the control variable (CV). Thrust control is typically accomplished indirectly and imprecisely, by controlling a variable that has a strong correlation with thrust, such as fan speed or corrected fan speed. ${ }^{11}$ However, direct control of an estimated thrust variable may soon become a reality due to advancements in onboard nonlinear engine modeling ${ }^{12,13}$, and model-based engine control. ${ }^{14,15}$ For this reason, and because it simplifies the control design necessary for dynamic systems analysis (DSA), it was considered acceptable to make this assumption and move forward with direct thrust control.

The motor control logic was then designed to run the steady-state motor power schedule from Figure 2, but with additional nonlinear logic to provide the maximum allowable (rated) power assist during acceleration transients. Further, the nonlinear logic also reduces the motor power applied during deceleration transients, where LPC stall margin is typically at its lowest. This means the motor transient logic may help reduce the requirements for a variable bleed valve (VBV) to protect LPC SM during deceleration, in addition to what it can do during steady-state. It is important to extract the maximum possible benefit for acceleration transients from the motor, as doing so will make the acceleration response time (performance) versus operability (HPC SM) as favorable as possible. Figure 4 shows a block diagram of the engine controller, containing both the fuel flow and motor control logic.

American Institute of Aeronautics and Astronautics 


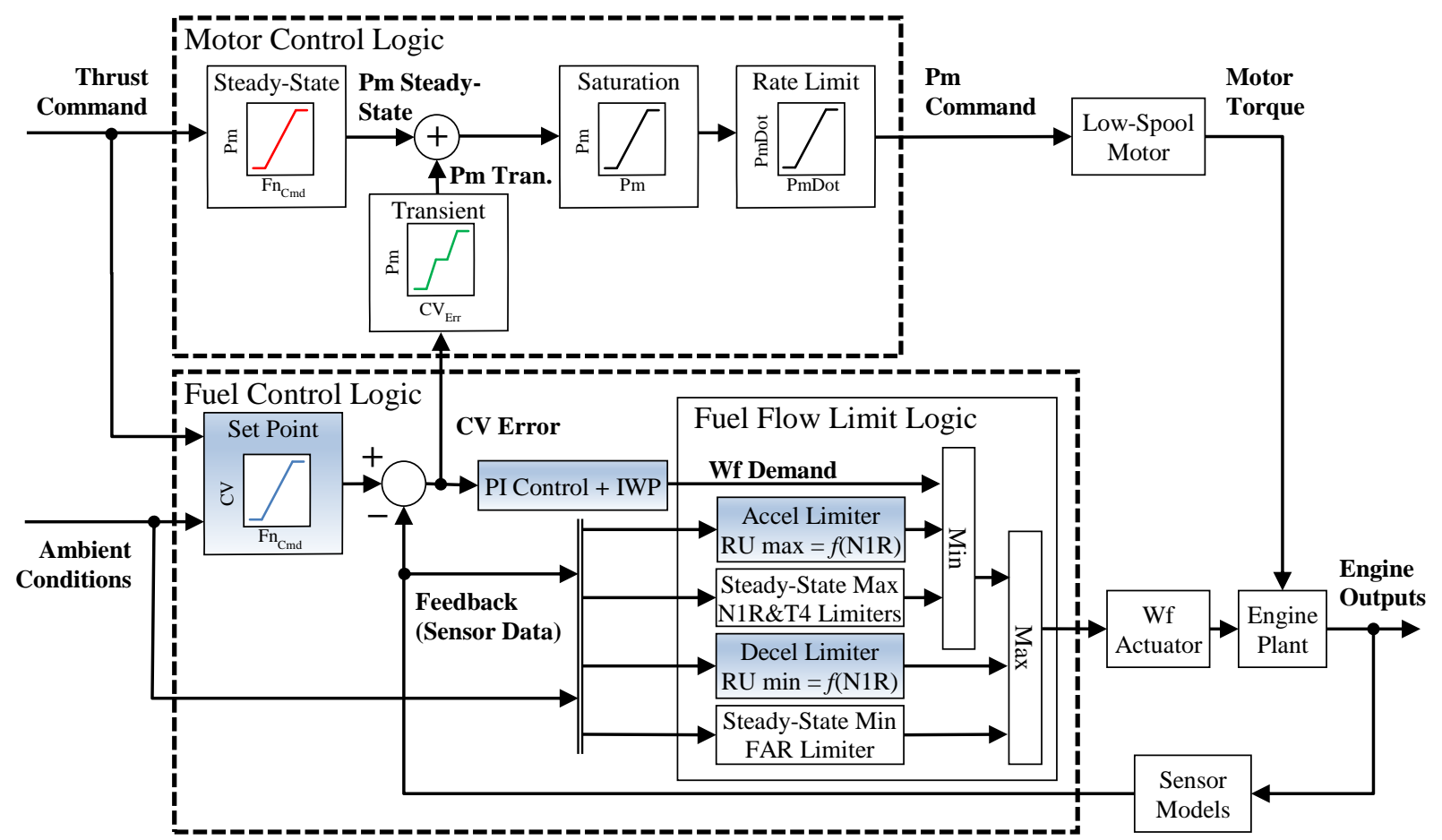

Figure 4: Thrust control logic for the closed-loop NASA hFan dynamic model.

The fuel flow control loop shown in the bottom half of Figure 4 is a modified TTECTrA control architecture, which normally contains a setpoint function, and a proportional-integral (PI) regulator with integral windup protection (IWP). It also contains limit logic to keep the engine operating within its design limits. This limit logic includes acceleration and deceleration limiters and $\mathrm{min} / \mathrm{max}$ selection logic to switch between nominal and limit control. These items are indicated in blue, as they are designed using TTECTrA. Added to this are steady-state minimum and maximum limit logic blocks, which protect maximum limits on corrected fan speed (N1R), HPT inlet temperature (T4), and a minimum limit on fuel/air ratio (FAR). TTECTrA does not include or design these, as they are not necessary for conducting dynamic systems analysis, and they can even have a deleterious effect on system response time. ${ }^{16}$ However, it was determined that they are useful, if not necessary, to ensure that engine operating limits are not violated at different points in the flight envelope. This architecture (the PI with min/max limit logic and bumpless transfer via IWP) is representative of time-tested control logic used in the aerospace industry. Thus it was considered by the authors that demonstration of successful flight (maintaining uncertainty stall margin stack-ups and meeting performance requirements) with this closed-loop system throughout the operating envelope is sufficient to say that the $\mathrm{hFan}$ engine design is operable (i.e., has adequate transient operability margin built-in).

The setpoint function block in the diagram is a setpoint lookup table that selects the correct value of the control variable to obtain the desired thrust setpoint value. In this case, it is a conversion from a normalized or fractional maximum thrust, to an actual desired thrust value in lbf. The PI controller is a gain scheduled design that selects or interpolates the designed PI gains as the system dynamics change given the engine's current state. The PI controller contains IWP logic to ensure the PI's integrator does not run away and cause unsafe operation while the closed-loop system rides a safety limit, which ensures bumpless transfer when switching control modes.

The acceleration or accel limiter block limits fuel flow such that transient operability variables are not violated during engine spool accelerations. Typically, the operability variables of interest for accelerations are HPC SM and T4. The accel limiter is based on ratio unit (RU) max values that are scheduled to the current altitude, Mach number, and current engine power level that is represented by N1R. The RU variable is the ratio, $W f$ divided by $P s 3$ (fuel flow per unit static HPC exit pressure), and is a variable that correlates with FAR. FAR is not sensed in typical engines, yet needs to be controlled carefully to prevent combustor flame-out and other issues.

The TTECTrA WfIPs3 max schedule design function is used to design accel limit schedules at various flight conditions, and these schedules are then placed in a large lookup table for interpolation during run-time operation. The design function produces these schedules from data obtained through open-loop acceleration simulations where the fuel flow is ramped up at varying rates until the transient operability margin constraints are just met. Then the WfIPs 3 data from these simulations can be used to construct a max WfIPs 3 schedule, which can be considered a sort

American Institute of Aeronautics and Astronautics 
of redline (a line separating acceptable and unacceptable closed-loop system states) that the control logic should ensure the engine never crosses.

The decel limiter block logic is designed using the TTECTrA WfIPs 3 min schedule function, and this design function works similarly to the Wf/Ps 3 max schedule function. However, the Wf/Ps 3 min limit is designed to limit the rate of engine deceleration, such that the variables relevant to decelerations (usually LPC SM, minimum FAR to protect against combustor blowout, and sometimes fan SM) are maintained above minimum acceptable values.

The novel part of the hFan control architecture is the motor control logic. This logic computes a motor control signal as the sum of a steady-state component (a function of thrust command as indicated in Figure 2), and a transient component (a function of the control variable error). This total motor control signal is then saturated to the minimum and maximum allowable power values that the motor can support. It also features a deadband characteristic to inhibit chattering, although the motor control logic is not overly prone to chattering due to the relatively small percentage of the total propulsion system power that the motor is in control of. These nonlinear characteristics make the controller act like a bang-bang or on/off controller, because, given the ad-hoc design parameters chosen for this transient motor control logic, as soon as the control variable error exceeds the deadband, the output power signal already hits the saturation limit. The initial hypothesis for the control design is that this is a simple and effective way to get the maximum assist torque out of the motor during transients, and so this was the control architecture chosen.

The motor input was supplied to the engine model as a negative horsepower extraction. No losses were included in the motor model, and the $1380 \mathrm{HP}$ motor power rating was taken to be the only constraint in the motor system. Therefore, the full maximum $1380 \mathrm{HP}$ was assumed to be deliverable to the motor. These are all simplifying assumptions, and were made given the fact that this work is exploratory in nature and there are still many unknowns. Further, losses are particularly important when assessing system-wide impact (i.e., block fuel burn for a given mission), and not necessarily so for capturing dynamic response. Since this work is mainly concerned with dynamic analysis and the impact transient operation can have on engine design constraints, the losses were considered to be outside the scope of this work and thus were neglected.

There may be some performance gains to be had by a more sophisticated control scheme, for instance, a multiinput, multi-output (MIMO) controller that simultaneously varies the fuel flow and motor power to track two independent outputs. However, this controller demonstrated suitable performance without a significant deviation in design from conventional control architectures used in the industry. This architecture was chosen for this reason. After designing the controller, it was integrated with the hFan engine plant, and simulation studies were conducted with the resulting closed-loop system to assess its operability throughout the flight envelope.

\section{B. Closed-Loop Simulation Test}

This subsection shows results from a Monte Carlo analysis where the engine is run through burst and chop transients at random flight conditions and with random minimum and maximum throttle settings (thrust setpoints). Engine transients are also run over a standard set of test conditions throughout the envelope; these conditions are the same ones that the NPSS steady-state model was run at, for purposes of testing the engine design. The set of test conditions the engine was run at, along with relevant transient results are shown in Figure 5.

These Monte Carlo tests were designed so that they include cases where the engine performs an acceleration at static (and near-static, $<0.1 \mathrm{Mach}$ ) conditions, starting from idle power settings (at or below $15 \%$ rated SLS thrust), and ending at the maximum power setting. These cases correspond to a test of the US Federal Aviation Administration requirement, specified in Title 14, Part 33.E, Section 33.73. This requirement is referred to in this paper as the "5second" requirement, and states that engines must be able to accelerate safely, without deleterious conditions such as stall or over-temperature, from no more than $15 \%$ thrust to $95 \%$ the maximum rated takeoff thrust within 5 seconds. ${ }^{17}$ In all of the flight conditions where the 5-second requirement might be tested (below 12,500 ft, and near static Mach), the engine accelerates within 5 seconds without violating operability constraints, and thus is considered to meet the performance requirement. Note that points where the engine controller parameters were designed and their associated performance data are marked with red crosses in Figure 5, whereas the random, Monte Carlo test cases are drawn with blue dots. Further, the flight envelope, or the set of flight conditions (altitudes and Mach numbers) the engine is designed to run at is indicated with thick, solid black lines in the top right subplot of Figure 5.

American Institute of Aeronautics and Astronautics 

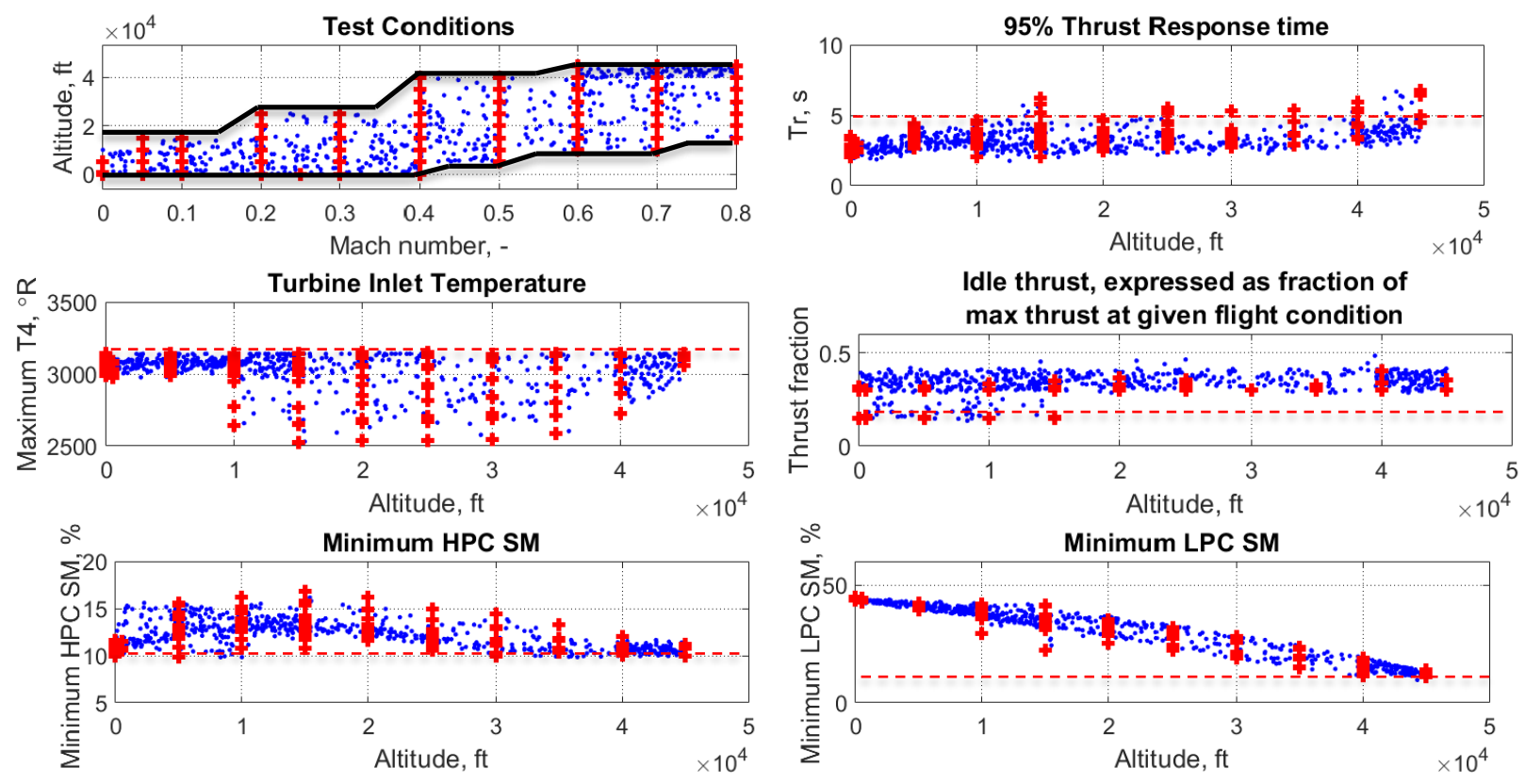

Figure 5: Scatter plots of the flight conditions tested in the Monte Carlo analysis, the $95 \%$ acceleration thrust response time, turbine inlet temperature, the idle thrust before starting the burst acceleration (expressed as a percentage of maximum), and minimum HPC and LPC SM. Blue dots represent random Monte Carlo test points, and red crosses represent results from running over a grid of standard flight conditions.

This figure shows that the $15 \%$-to-95\% time response for idle to full power acceleration transients (response time, or $\operatorname{Tr}$ in top right) is consistently made in less than 5 seconds for all altitudes below about $12,000 \mathrm{ft}$. This means the closed-loop system meets the performance (thrust response) requirements. Further, the maximum turbine inlet temperature (or T4 at middle left) of 3140 degrees Rankine is only violated in 3 of the test cases (and by a maximum magnitude of 10 degrees). The system could be designed to protect this limit in all cases if the controller is designed to be slightly less aggressive (slower response) in the neighborhood of those points or if the T4 maximum value for the limit logic is reduced slightly. In addition, the idle thrust percentage (middle right) is shown to be an average of about 30 to $40 \%$ of the maximum thrust, however, it is at or below $15 \%$ for several of the cases below $15,000 \mathrm{ft}$. These cases correspond to flight Mach numbers below 0.1. Here, the aircraft is considered to be on the ground and thus the engine is starting the transient from a ground idle power condition as opposed to a higher, flight idle condition. Note that this idle thrust percentage is computed as a percentage of the maximum thrust attainable at the current flight condition, and maximum attainable thrust will decrease as the engine runs at higher Mach numbers and altitudes.

Lastly, the minimum HPC and LPC stall margins (bottom left and right) in these simulations are above $10 \%$ for over $97 \%$ of the test cases. In the cases where the $10 \%$ SM constraints are violated, they are violated by a half percent or less. These violations can be corrected by making the WfIPs3 accel and decel limit schedules more conservative, and thus the engine response slightly slower.

This study shows that, for a flight envelope spanned by the test conditions attempted (top left), the engine can be considered operable. This is because a control system can be designed for it, such that the closed-loop system meets performance requirements while not exceeding operability constraints. It is worth noting that this study was performed on the engine running at a nominal health condition. Future work should account for uncertainty in engine health parameters as well, to ensure that the closed loop system is operable throughout the envelope and for all health conditions.

\section{Dynamic Systems Analysis Trade Studies}

Given that the hFan engine design is operable throughout the flight envelope, an assessment of the operability margins built into the engine should be conducted. If the margins are conservative, the steady-state constraints in the engine design process may be relaxed, or the engine design variables may otherwise be made more aggressive. This may open up the engine design space such that it includes additional, more efficient design solutions. For instance, these solutions may include engines with fewer compression stages, but are more highly loaded, such that the engine

American Institute of Aeronautics and Astronautics 
achieves the same overall pressure ratio with less weight, but runs with lower design stall margins. Tight propulsion system control can then ensure that stall margin constraints and performance requirements are met, without the need for excess margin.

As mentioned previously, TTECTrA is a suite of tools made for conducting transient analyses on turbofan engines. Having developed a baseline control system for the hFan in Section IV, a DSA may be conducted on the hFan design. This is done by varying the operability design constraints for the control system, and observing the effect on performance obtained when designing controllers with these different constraints. There is a tendency for engines to run to lower and lower operability margin (i.e., stall margin) values as the engine accelerates or decelerates faster and faster. A DSA study can quantify the tradeoffs between performance variables such as acceleration time (rise time) and operability variables such as HPC SM.

To set up the hFan for DSA, the baseline controller described in Section IV was taken, and the acceleration limit logic (Wf/Ps3 max schedule) was designed for 6 different HPC SM constraint values to observe how performance varies as the engine protects different SM constraints. The steady-state $W f$ limit logic was removed to ensure that it did not affect the results. Then, a 15\% to 100\% max thrust acceleration transient was run with each controller at SLS on a hot day $\left(27^{\circ} \mathrm{R}\right.$ above ISA temperature), to observe how performance and operability vary during the transient. The 15-to-95\% acceleration response time vs HPC SM and max T4 are shown in Figure 6, along with traces of the thrust, HPC SM, and T4 simulation data.
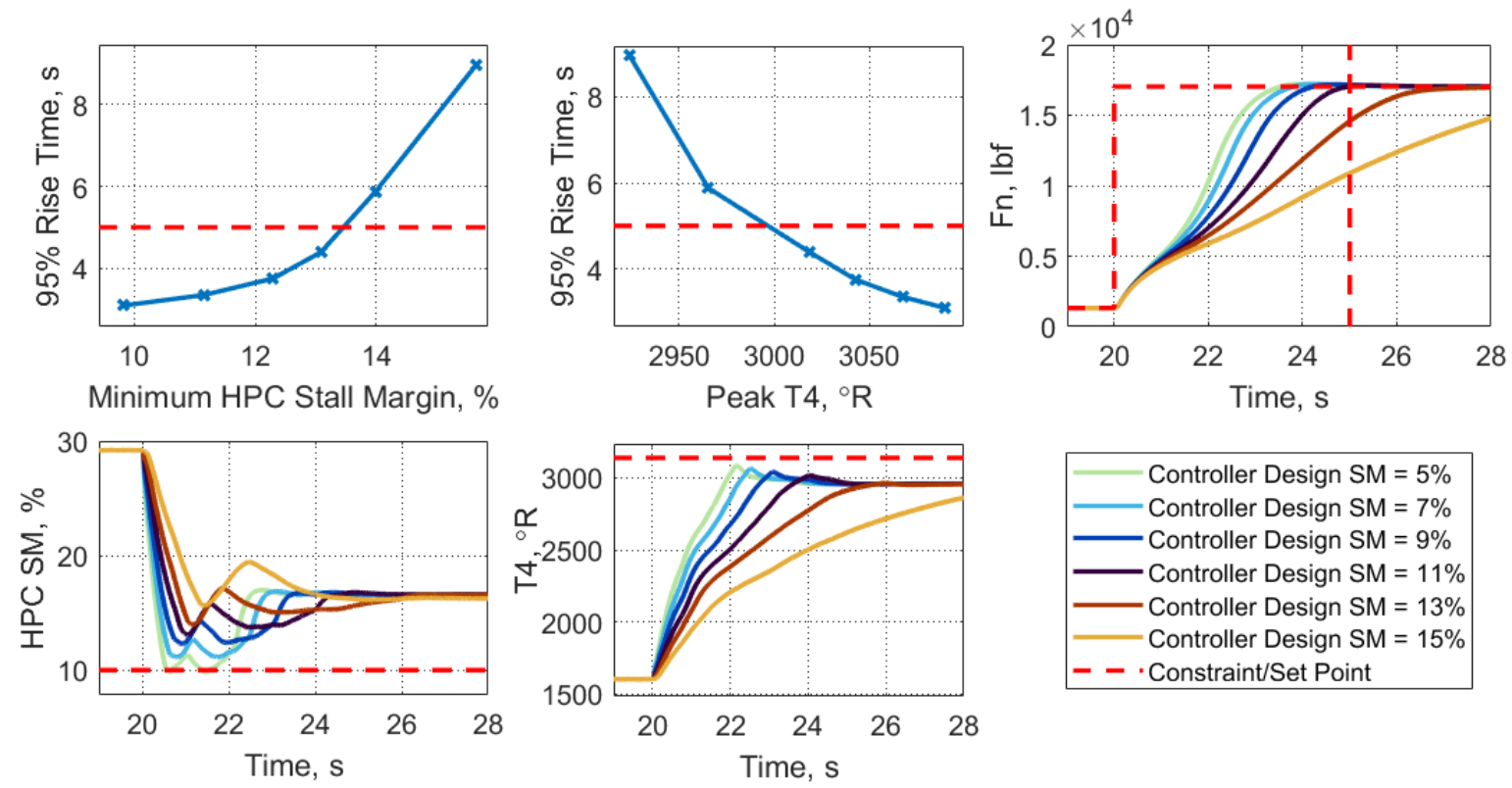

Figure 6: Results of a dynamic systems analysis on the baseline hFan closed-loop system.

In Figure 6, the top left and top middle subplots show DSA metrics (minimum HPC SM and maximum T4 plotted vs 95 percent rise time, observed when running with the different controller designs) captured from the transient simulation data shown in the remaining three subplots. The data in this figure show that the five second response requirement (accelerating faster than 5 seconds while not exceeding the $10 \%$ transient stack) is satisfied by the four cases where min HPC SM is designed for 5, 7, 9, and $11 \%$, but not for the $13 \%$ and $15 \%$ cases. Further, note that the actual minimum HPC SM values exhibited by the engine during these transients are all above the design values. This is the intended controller behavior, because the controller should not violate its operability constraints under any circumstances. The minimum HPC SM responses observed when doing a 15-to-100\% thrust transient are typically one or more percent above the design value for a typical transient; the minimum HPC SM will run much closer to the constraint value when starting the transient from a lower thrust, closer to the minimum ground idle thrust value (which, in the $\mathrm{hFan}$, is between 8 and $9 \%$ rated thrust).

Looking at the top-left subplot in Figure 6, the engine will perform a 5 second acceleration running somewhere between $13 \%$ and $14 \%$ minimum HPC SM. This suggests that there may be at least 3\% HPC SM more than necessary built into the engine design, given this controller architecture and assuming that the minimum HPC SM needed for the uncertainty stack is $10 \%$. Note that it is likely that as the engine deteriorates or the engine runs at different flight 
conditions, the engine may run a transient HPC SM lower than $13 \%$ for a 5 second acceleration, and so the $3 \%$ HPC SM reduction recommendation may be too aggressive. Therefore, future work should incorporate uncertainty analysis to cover more engine health conditions and flight conditions to obtain a more confident recommendation for redesign.

In addition to examining the performance-operability trend for the baseline closed-loop system, it is also useful to observe how this performance-operability trend varies as the electrical system parameters (motor power magnitude and ramp rate limit) change. The effect of these parameters on transient system tradeoffs are not well known. One hypothesis for the control design is that it is desirable to apply a steep ramp rate causing the low spool motor power to quickly reach its maximum level during acceleration transients. To test this hypothesis, engine transient response was evaluated for several different motor applied power ramp rates. To accomplish this, the ramp rate parameter for the "rate limit" block in Figure 4 was varied to achieve motor power rise time targets of $0.15 \mathrm{~s}, 1 \mathrm{~s}, 5 \mathrm{~s}, 10 \mathrm{~s}$, and no use of the motor at all (indicated as inf s). Note that these ramp rate values were run with each of the six different fuel flow controller designs described above, so that the engine's performance and operability can be characterized for each combination of these control design variables. The results of this study are shown in Figure 7.

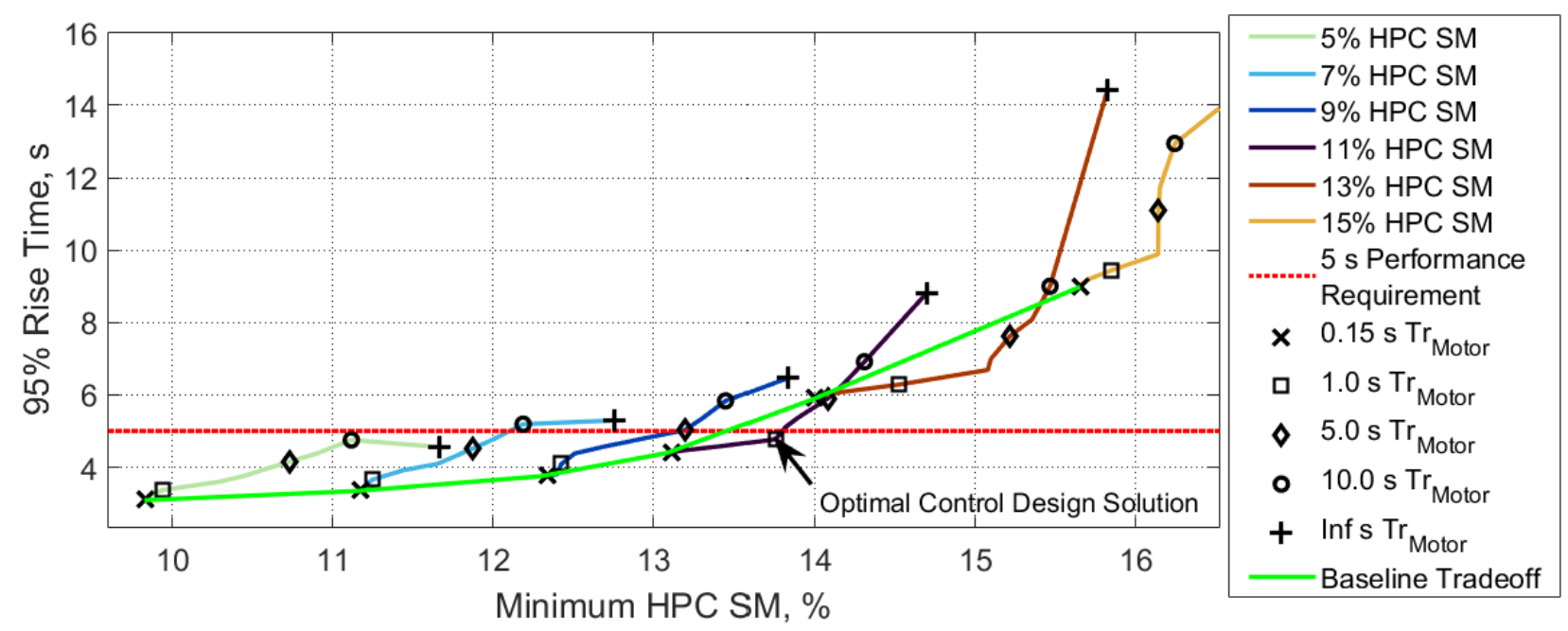

Figure 7: Acceleration response time versus minimum HPC SM trades obtained when acceleration limiter design and motor power ramp rate are varied simultaneously. Each colored line represents the effect of varying motor ramp rate while holding the acceleration limiter constant, and the dashed green line represents the baseline tradeoff (with the nearly instantaneous motor ramp rate).

The green line in Figure 7 connects data points representing the 95\% rise time across each of the six fuel flow controller designs when using the baseline motor ramp rate of 0.15 seconds, or almost instantaneous ramp rate. Applying this motor ramp rate achieves a sub-optimal performance-operability trade (meaning that it does not achieve the highest possible minimum HPC SM for a 5 second acceleration). The case where the motor does not turn on during the transient (infinite motor rise time represented by the '+' markers in the figure) is also sub-optimal. The best trade seen in this data set is obtained with a ramp rate such that the motor power rise time is around 1 second, and specifically, when running with the 11\% HPC SM controller design. This optimal design is indicated in Figure 7 with an arrow, labeled "optimal control design solution." Figure 7 shows that, for any of the six fuel control designs, decreasing the motor ramp rate (moving up and to the right, starting from the baseline tradeoff line) will increase acceleration time but also increase the minimum HPC SM. In some cases (specifically, with fuel controllers designed for $11 \%$ or greater HPC SM), the minimum HPC SM increases faster than response time, and thus a better tradeoff can be obtained with a slower motor ramp rate. This data shows that instantaneously applied motor power is not generally optimal (the initial hypothesis is rejected), and that there should be some acceleration limiter on the motor power command, whether it is a ramp rate limit or some other kind of limit.

Further, it was also hypothesized that a motor designed for a peak power magnitude greater than the nominal design power might improve the performance-operability trade. To investigate this hypothesis, the same study above (running all six accel limiter designs with different motor ramp rates) was performed while running with six different maximum motor power constraints. These different constraints allow the transient motor control logic to apply additional assist power. This is because, for acceleration transients running to the maximum power level or thrust setpoint, the steady-state motor command at max power is up against the maximum motor power constraint. Therefore,

American Institute of Aeronautics and Astronautics 
the motor cannot provide any additional assist power during the transient. However, it is worth noting that acceleration transients are short (on the order of seconds), and a realistic motor's maximum peak power rating may be significantly higher than its continuous (steady-state) power rating. Given that the studies above run the motor to its steady-state maximum value and not necessarily to a maximum peak power, it may be that these studies do not represent the full benefits that a motor can provide for transient assist type operation. Given this fact, the constraint for motor power was varied to six different values $(1.00,1.25,1.50,1.75$, and 2.00 times the nominal maximum motor power rating). A subset of the data from this study (for 11\% and 13\% HPC SM acceleration limiter designs only) is shown in Figure 8.

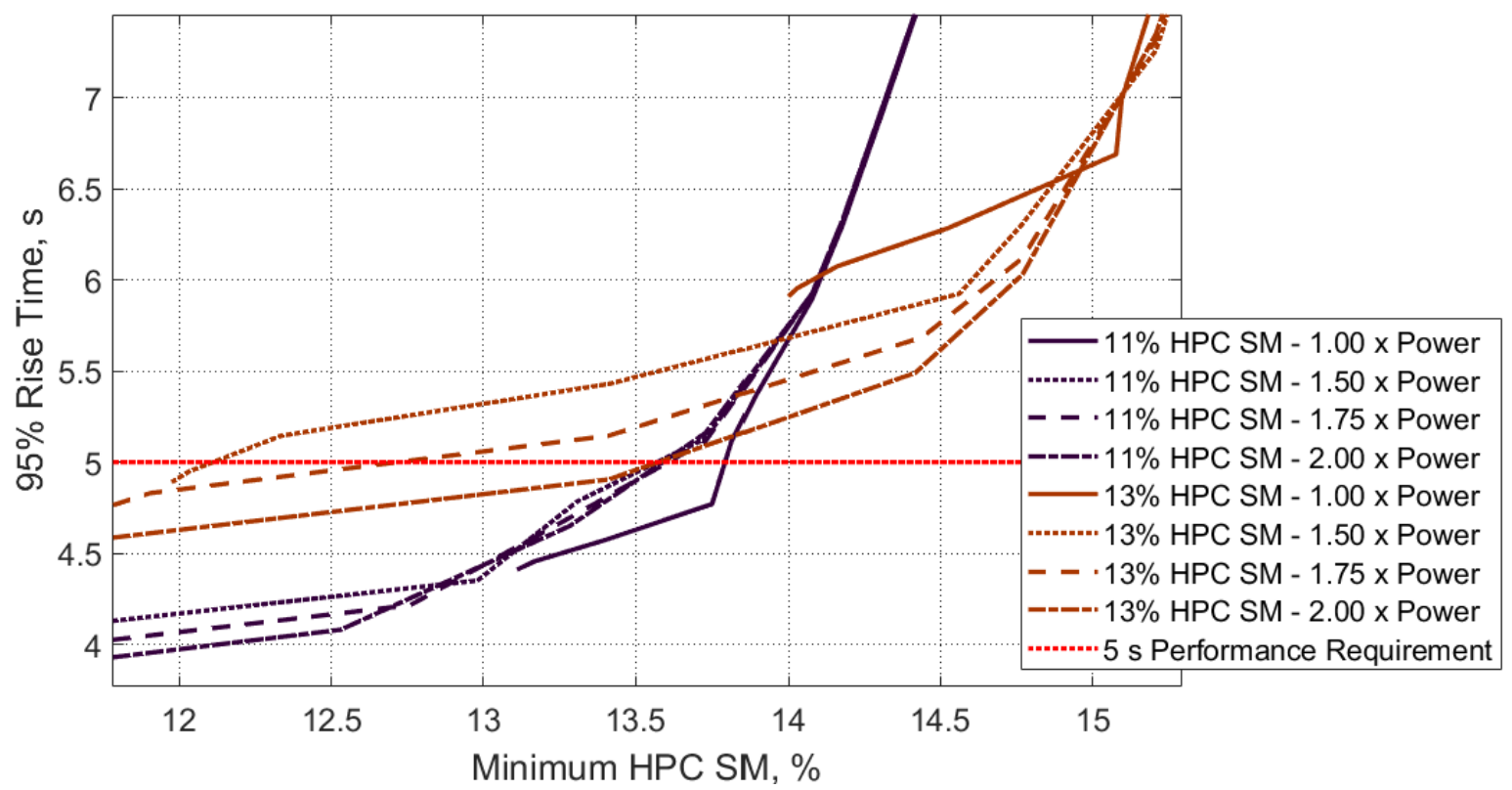

Figure 8: Acceleration response time vs minimum HPC SM run with different maximum transient power constraints. Each line connects the response times and SMs from runs with the same acceleration limiter design and same maximum motor power, but with varying motor power ramp rates.

The trend we have observed from this data is that, for the faster acceleration schedule in the fuel flow controller (i.e., the 11\% HPC SM data), the motor power magnitude does not affect the performance-operability trade significantly. This can be seen because the 11\% HPC SM curves for different power constraints are similar. Instead, the ramp rate is the primary driver of the trend. However, for the slower acceleration limiter design (the 13\% HPC SM data), increasing the power constraint affects the trade significantly. Specifically, it decreases both the response time and minimum HPC SM obtained with the fast ramp rates. Note that the response time improvement given by significantly more motor power applied during the accel with a slower fuel controller (see 13\% SM design with two times nominal power) is not enough to achieve higher minimum SM for 5 second accelerations compared to the 11\% SM control designs. However, it is possible that power constraints above two times nominal (e.g. three or four times), used with slower fuel flow controller designs, may be able to achieve higher minimum SM for 5 second accelerations compared to controllers running with nominal power. For instance, if one were to extrapolate the trend of the brown, 13\% SM data in Figure 8 to higher powers, it is conceivable that, at some higher motor power level, the engine should be able to do a 5 second acceleration with $14 \%$ or greater minimum SM. However, these power constraints above two times nominal were not examined in this study, as they were seen as too far of a departure from the baseline hFan configuration.

Note that the maximum improvement in minimum HPC SM obtained for $5 \mathrm{~s}$ accelerations using any of the control designs examined in this study is no more than a half percent SM improvement compared to the best solution from Figure $6(13 \%-14 \%)$. Since this is a meager improvement, it was desired to determine if motor operation may be able to make the trade space more favorable between acceleration response time performance and other engine performance variables such as maximum turbine inlet temperature. Figure 9 shows the response time versus peak $T 4$ from the same control design solutions examined in the previous figure.

American Institute of Aeronautics and Astronautics 


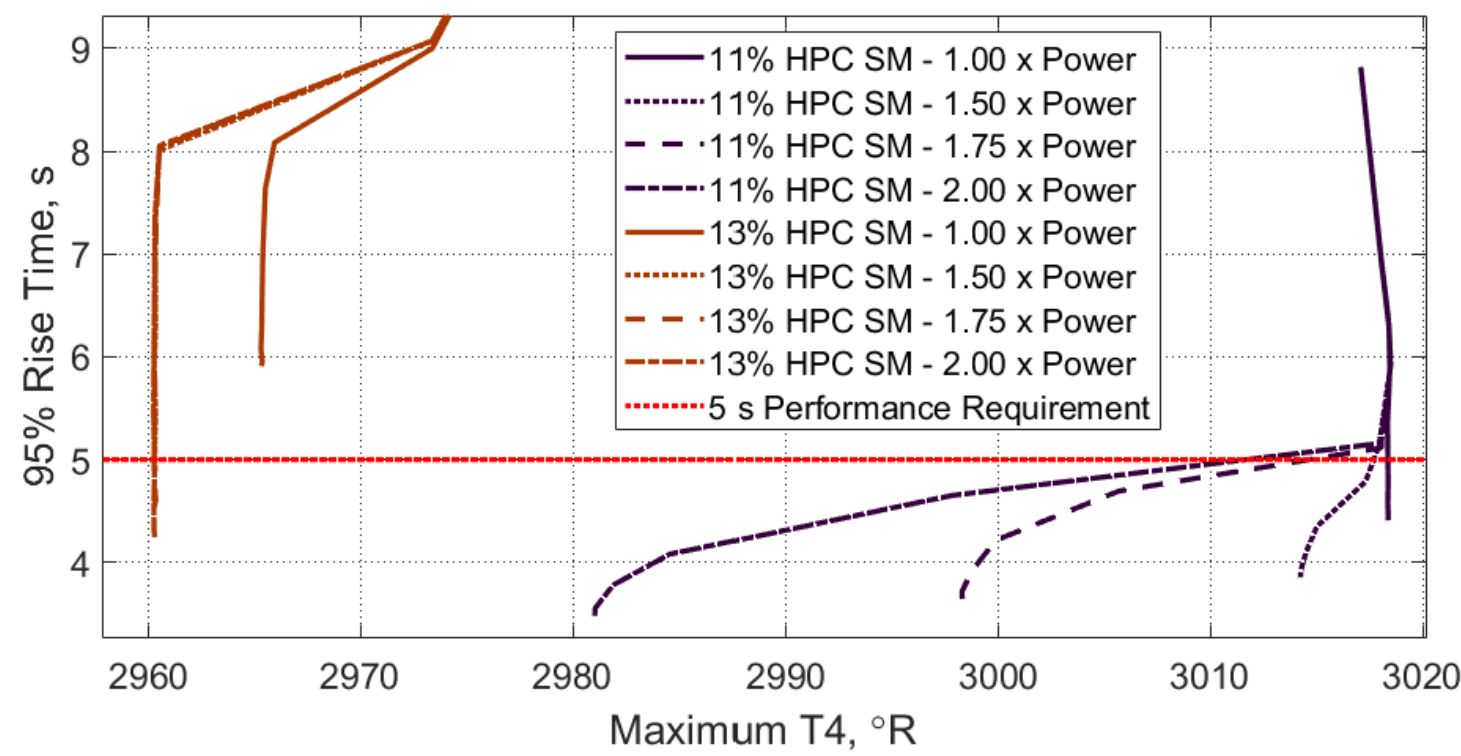

Figure 9: Acceleration response time vs maximum turbine inlet temperature (T4) run with different maximum transient power constraints. Each line connects the response times and SMs from runs with the same acceleration limiter design and same maximum motor power, but varying motor power ramp rates.

This figure shows that with slower acceleration limiter designs (the brown, 13\% HPC SM data), increasing the motor ramp rate decreases the response time without significantly affecting the max $T 4$ and thus improves the response time vs $T 4$ tradeoff. However, with faster acceleration limiter designs (the dark purple, 11\% HPC SM data), the increased motor power tends to lower $T 4$ and response time when the ramp rate is fast. Maximum $T 4$ is reduced over $35^{\circ} \mathrm{R}$ comparing the most aggressive control solution (11\% HPC SM acceleration limiter, maximum motor ramp rate, and 2 times nominal power constraint) to any of the control solutions with the nominal motor power constraint. On the other hand, with lower power constraints, the motor power ramp rate affects response time without significantly affecting maximum $T 4$, which suggests that fast motor response with a high magnitude is required to achieve transient $T 4$ reduction using the motor.

The conclusion to be drawn from the motor control trade studies illustrated via Figure 7, Figure 8, and Figure 9 is that the most favorable motor operation for HPC SM is a relatively fast, but not instantaneous motor power ramp rate. The trend in Figure 8 shows that if a relatively slow fuel flow acceleration limiter is used (the brown 13\% HPC SM data), and the transient motor power is increased, this will cause the engine to accelerate faster (lower rise time) for the same minimum HPC stall margin. If one extrapolates this trend to even higher power constraints, it is likely that one could find better control design solutions in terms of performance-operability trade, compared to the most optimal solution found in this work. Future work includes testing this hypothesis in simulation with the hFan. Further, the results explored in this section suggest that applying a high magnitude of motor power and ramping it up as quickly as possible during the acceleration will improve the performance vs maximum $T 4$ trade space. Therefore, future studies should first consider how the controller should best be designed to open up the engine design space to more efficient design solutions.

One strategy to extract the maximum benefit from the motor is to redesign the engine with better efficiency via a more aggressive HPC op-line and a smaller, appropriately sized transient stack built into the steady-state HPC SM. Then the fuel flow and motor controllers should be designed to protect as much HPC SM as possible during acceleration while still allowing the engine to meet the 5 second acceleration time requirement. An alternative strategy may be to design the controller to limit the transient $T 4$ as much as possible, and treat the improvement of HPC SM as a secondary objective. Then the engine could be resized or redesigned with reduced HPT cooling bleed flows such that it can be more efficient. These strategies and potentially others should be explored further and compared to find optimal hybrid gas turbine engine designs.

American Institute of Aeronautics and Astronautics 


\section{Summary and Conclusions}

This paper described the NASA hFan: A Numerical Propulsion System Simulation (NPSS) model of a 17,000 lbf dual spool turbofan engine with a 1380 horsepower electric machine mounted on the low spool shaft. This engine model was integrated into the MATLAB/Simulink environment via the NPSS S-Function, and then a controller was designed around it. Transient simulation tests were conducted with the closed-loop system throughout the flight envelope to ensure the system satisfies performance requirements while not exceeding operability constraints. The results of these tests show that the closed-loop system is able to maintain the operability of the engine throughout the flight envelope. This demonstrates that the engine is capable of acceptable performance throughout the envelope, provided it is appropriately controlled. Further, a dynamic systems analysis (DSA) was performed at the sea-level static (SLS) flight condition to make recommendations about the steady-state high pressure compressor (HPC) stall margin (SM) design value. It was found that the steady-state HPC SM constraint may be reduced by about 3\% to enable a wider engine design space that may contain more efficient designs. Lastly, a set of trade studies varying the control design for the electric motor were performed to assess how motor operation affects the performance-operability tradeoff. It was found that a rate limiter could be added to the motor input signal to improve the HPC SM for a 5 second acceleration by as much as a half percent of margin. Further, it was found that increasing the motor power constraint (allowing the transient motor control logic to apply a higher motor power value during the transient) and not limiting the motor signal rate may allow more than $35^{\circ} \mathrm{R}$ turbine inlet temperature (T4) reduction. In the future, it may be advantageous to consider applying a more sophisticated algorithm to filter the motor power signal (e.g.: an acceleration limiter) to get better HPC SM improvement than can be obtained with the rate limiter. In addition to these specific findings, some general trends can be concluded from the trade studies. First, it is shown that motor response rate and maximum applied motor power both factor into dynamic response, and secondly, combined fuel and motor power control design should be conducted to achieve optimal overall performance.

\section{Acknowledgement}

Thanks go to William Haller of NASA Glenn Research Center (GRC) for guiding and managing this work, and to Sanjay Garg, also of NASA GRC for his useful input and suggestions toward studying the hFan system. The authors also thank Thomas Lavelle of NASA GRC for his assistance developing transient simulation capability for the hFan. Further, the authors would like to thank the NASA Advanced Air Transport Technologies Project for funding and supporting this work.

\section{References}

${ }^{1}$ Cao, W., Mecrow, B., Atkinson, G., and Bennett, J., "Overview of Electric Motor Technologies Used for More Electric Aircraft (MEA)," IEEE Transactions on Industrial Electronics, Vol 59, no 9, pp 3523-3531, September 2012. ${ }^{2}$ Ashcraft, S.W., Padron, A.S., Pascioni, K.A, Stout Jr., G.W., and Huff, D.L., "Review of Propulsion Technologies for N+3 Subsonic Vehicle Concepts," NASA TM 2011-217239, October, 2011.

${ }^{3}$ Hathaway, M.D., Del Rosario, R., and Madavan, N.K., "NASA Fixed Wing Project Propulsion Research and Technology Development Activities to Reduce Thrust Specific Energy Consumption," NASA TM 2013-216548, July 2013.

${ }^{4}$ Antcliff, K.R., Guynn, M.D., Marien, T.V., Wells, D.P.., Schneider, S.J., and Tong, M.T., "Mission Analysis and Aircraft Sizing of Hybrid-Electric Regional Aircraft," AIAA 2016-1028, AIAA SciTech, 54 ${ }^{\text {th }}$ AIAA Aerospace Sciences Meeting, San Diego, CA, January 4-8, 2016.

${ }^{5}$ Claus, R.W., Evans, A.L., Lylte, J.K. and Nichols, L.D., "Numerical Propulsion System Simulation," Computing Systems in Engineering, Vol 2, no 4, pp 357-364, 1991.

${ }^{6}$ Chin, J.C., Csank, J.T., Haller, W.J., and Seidel, J.A., "An Introduction to Transient Engine Applications Using the Numerical Propulsion System Simulation (NPSS) and MATLAB,” NASA, NASA/TM 2016-218922, January, 2016.

${ }^{7}$ Bradley, M. K., Droney, C. K., "Subsonic Ultra Green Aircraft Research: Phase II - Volume II - Hybrid Electric Design Exploration," NASA CR-218704. 2015.

${ }^{8}$ Csank, J.T., and Thomas, G.L., "Dynamic Analysis for a Geared Turbofan Engine with Variable Area Fan Nozzle," AIAA 2017-4819, 53 ${ }^{\text {rd }}$ AIAA/ASME/SAE/ASEE Joint Propulsion Conference, Atlanta, GA, July 10-12, 2014.

${ }^{9}$ Csank, J.T., and Zinnecker, A.M., "Application of the Tool for Turbine Engine Closed-loop Transient Analysis (TTECTrA) for Dynamic Systems Analysis," AIAA 2014-3975, 50 ${ }^{\text {th }}$ AIAA/ASME/SAE/ASEE Joint Propulsion Conference, Cleveland, OH, July 28-30, 2014.

American Institute of Aeronautics and Astronautics 
${ }^{10}$ Zinnecker, A.M., and Csank, J.T., "A Methodology to Assess the Capability of Engine Designs to Meet Closedloop Performance and Operability Requirements," AIAA 2015-4023, 51 $1^{\text {st }}$ AIAA/SAE/ASEE Joint Propulsion Conference, Orlando, FL, July 27-29, 2015.

${ }^{11}$ Csank, J.T., May, R.D., Litt, J.S., and Guo, T.-H., "Control Design for a Generic Commercial Aircraft Engine," AIAA 2010-6629, $46^{\text {th }}$ AIAA/ASME/SAE/ASEE Joint Propulsion Conference and Exhibit, Nashville, TN, July 2528, 2010.

${ }^{12}$ Orme, J.S., and Schkolnik, G. S., "Flight Assessment of the Onboard Propulsion System Model for the Performance Seeking Control Algorithm on an F-15 Aircraft," NASA TM-4705. 1995.

${ }^{13}$ Henriksson, M., Grönsted, T., and Breitholtz, C., "Model-Based On-Board Turbofan Thrust Estimation," Control Engineering Practice, Vol 19, no 6, pp 602-610, 2011.

${ }^{14}$ Connolly, J.W., Csank, J.T., Chicatelli, A., and Kilver, J., "Model-Based Control of a Nonlinear Aircraft Engine Simulation using an Optimal Tuner Kalman Filter Approach," AIAA 2013-4002, 49 ${ }^{\text {th }}$ AIAA/ASME/SAE/ASEE Joint Propulsion Conference, San Jose, CA, July 14-17, 2013.

${ }^{15}$ Csank, J.T, and Connolly, J.W., "Model-Based Engine Control Architecture with an Extended Kalman Filter," AIAA 2016-1623, AIAA SciTech, AIAA Guidance, Navigation, and Control Conference, San Diego, CA, January 48, 2016.

${ }^{16}$ May, R.D., and Garg, S., "Reducing Conservatism in Aircraft Engine Response Using Conditionally Active MinMax Limit Regulators,” NASA TM-217814. 2012.

${ }^{17}$ Federal Aviation Administration, "Title 14 of the Code of Federal Regulations", http://www.ecfr.gov/cgi-bin/text-

idx?SID=aa3a99819fbf162f142eee0ec759f8df\&mc=true\&node=se14.1.33 173\&rgn=div8, accessed April, 2018 